\title{
Research Paper \\ Prevalence of Risk Factors for Falls Among the Elderly Receiving Care at Home
}

\author{
Faroogh Na'emani ${ }^{1,2} \odot$, Morad Esmaiil Zali ${ }^{1,2} \odot$, Zahra Sohrabi ${ }^{2,3} \odot,{ }^{*}$ Ahmad Fayaz-Bakhsh ${ }^{4,5}$
}

1. Department of Health Management and Economics, School of Public Health, Tehran University of Medical Sciences, Tehran, Iran.

2. Research Group of Lifestyle \& Health Management at Home, Academic Center for Education, Culture and Research (ACECR), Tehran University of Medical Sciences, Tehran, Iran.

3. Department of Health Economics, School of Health Management and Information Sciences, Iran University of Medical Sciences, Tehran, Iran.

4. Sina Trauma \& Surgery Research Center, Tehran University of Medical Sciences, Tehran, Iran.

5. Department of Health in Disaster and Emergencies, School of Public Health, Tehran University of Medical Sciences, Tehran, Iran.

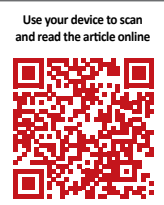

Crtation Na'emani F, Esmaiil Zali M, Sohrabi Z, Fayaz-Bakhsh A. [Prevalence of Risk Factors for Falls Among the Elderly Receiving Care at Home (Persian)]. Salmand: Iranian Journal of Ageing. 2019; 13(Special Issue):638-651. https://doi.org/10.32598/ SIJA.13.Special-Issue. 638

\section{https://doi.org/10.32598/SIJA.13.Special-Issue.638}

Key words:

Falling, Risk factors, Elderly, Homecare, Iran

\section{A B S T R A C T}

Received: 15 Sep 2018 Accepted: 05 Feb 2019 Available Online: 10 Mar 2019
Objectives Elderly people are at the highest risk for falling. In order to design and implement effective interventions and reduce the incidence of falling and its resulting injuries, the relative importance of each risk factor should be determined. This study aimed to determine how frequently different factors contribute to falls among the elderly served by one home health agency in Tehran.

Methods \& Materials This study was a descriptive-analytic and cross-sectional study. A total of 400 elderly were selected through random cluster sampling method from all 22 districts of Tehran City, Iran. The study data were collected through a researcher-made questionnaire of falling risk factors. The questionnaire consisted of two parts: first, demographic questions and second, questions related to falling in 7 dimensions (including 60 questions). The obtained data were analyzed in SPSS (V. 19) through descriptive and inferential statistics.

Results In this study, female samples were $52.5 \%$ of the participants $(n=400)$ and the elderly mean \pm SD age was $78 \pm 8$ years. The total frequency of falling among the elderly was $28 \%$ ( 112 out of 400 ). Among the elderly who had fallen $(n=112), 64(57.1 \%)$ of them were female and their Mean $\pm S D$ age was $80 \pm 7.5$ years. About $82 \%$ of them fell down inside their homes, with these separate frequencies: bathroom (19.6\%), toilet $(18 \%)$, bedroom $(18 \%)$, and hall $(16 \%)$. Parts of the body which were more frequently affected by falls in forms of injuries, bone fractures, dislocations, etc., were the pelvis (26.8\%), arms (22.3\%) and legs (19.6\%). Around $43.8 \%$ of the falling among the elderly had led to hospitalization, $44.9 \%$, between 1 and 5 days, $36.7 \%$ between 6 and 10 days, and $18.4 \%$ between 11 and 15 days. The relationship between falls and the medical, lifestyle, or environmental risk factors was non-significant.

Conclusion A high percentage of affected elderly were hospitalized after the incident, and among them a high percentage stayed in the hospital for a long time imposing considerable costs to the health system. Moreover, sensory and neuromuscular risk factors were the most frequent causes of falls which could be prevented by rehabilitation measures. Results showed that people who are afraid of falling have a higher risk of falling which requires adequate and special attention to this psychological risk factor.

\section{* Corresponding Author: \\ Ahmad Fayaz-Bakhsh, PhD.}

Address: Sina Trauma \& Surgery Research Center, Tehran University of Medical Sciences, Tehran, Iran.

Tel: +98 (912) 3277218

E-mail: fayaz@tums.ac.ir 


\section{Extended Abstract}

\section{Objectives}

F

alls reduce the quality of life and increase the cost of health care [1,2]. Approximately $30 \%$ of people aged 65 years or older experience at least one fall a year and the rate of hospitalization due to falling was reported to be $81 \%$

[3-6]. Falls in the elderly imposes heavy costs on society [7]. In Iran, there are no accurate statistics of the cost of treatment of falling injuries $[8,9]$. The average cost of hospitalization and post-mortem treatment for each elderly in Ireland is $\$ 6000$ [10] and in the United States $\$ 17000$ [11]. The most common social and psychological result of falling is the fear of falling [12]. According to studies, the daily activity of the elderly decreases after the experience of falling $[10,13]$. This study aims to investigate the prevalence of risk factors to falls among the elderly receiving care at home from Jihad Daneshgahi Specialized Center for inhome care services (called DAM) in Iran.

\section{Methods \& Materials}

This is a descriptive analytical study with a cross-sectional design. The study population consists of all elderly people living in Tehran aged over 65 years $(\mathrm{n}=20000)$ who received treatment from the DAM center in 2017. For sampling, the stratified sampling method was used. In this regard, 22 districts of Tehran were considered as strata. In each district, sampling was conducted. Using Cochran's formula, the sample size was determined as 400 . The inclusion criteria were 65 years or older and willingness to participate in the study. By searching in literature, at first 40 risk factors of falling were identified. Then, the most common factors were extracted. On this basis, a questionnaire was designed with 125 items in 7 dimensions.

Comments of the expert panel were used for testing the validity of the items. Only 60 items were verified by them. To test the tool reliability, the designed questionnaire was sent to 20 participants of the study two times with a 10-day interval. Results reported an alpha coefficient of more than

Table 1. The relationship between falling and its risk factors in study participants

\begin{tabular}{|c|c|c|c|c|c|}
\hline \multirow{3}{*}{ Risk Factors } & \multirow{3}{*}{ Variable } & & \multicolumn{2}{|c|}{ History of Falling } & \multirow{3}{*}{$\mathbf{P}$} \\
\hline & & & Yes & No & \\
\hline & & \multicolumn{3}{|c|}{ No. (\%) } & \\
\hline \multirow{6}{*}{ Psychological } & \multirow{2}{*}{ Urinary incontinence } & Yes & $63(56.3)$ & $105(36.5)$ & \multirow{2}{*}{0.012} \\
\hline & & No & 49(43.7) & $183(63.5)$ & \\
\hline & \multirow{2}{*}{ Sleep disorders } & Yes & $74(66.1)$ & $127(44.1)$ & \multirow{2}{*}{0.003} \\
\hline & & No & $38(33.9)$ & $161(55.9)$ & \\
\hline & \multirow{2}{*}{ Fear of falling } & Yes & $90(80.3)$ & $108(62.5)$ & \multirow{2}{*}{0.001} \\
\hline & & No & $22(19.7)$ & $180(37.5)$ & \\
\hline \multirow{2}{*}{ Medical } & \multirow{2}{*}{ History of surgery } & Yes & $72(64.3)$ & $158(54.9)$ & \multirow{2}{*}{0.037} \\
\hline & & No & $40(35.7)$ & $130(45.1)$ & \\
\hline \multirow{11}{*}{ Sensory-motor } & \multirow{2}{*}{ Walking with difficulty } & Yes & $83(74.1)$ & $119(41.3)$ & \multirow[t]{2}{*}{0.001} \\
\hline & & No & $29(25.9)$ & $169(58.7)$ & \\
\hline & \multirow{2}{*}{ Shaky hand/feet } & Yes & $61(54.5)$ & $95(33)$ & \multirow{2}{*}{0.008} \\
\hline & & No & $51(45.5)$ & 193(67) & \\
\hline & \multirow{2}{*}{ Knee pain } & Yes & $96(85.7)$ & $132(45.8)$ & \multirow{2}{*}{0.001} \\
\hline & & No & $16(14.3)$ & $156(54.2)$ & \\
\hline & \multirow{3}{*}{ Using mobility aids } & Yes & $81(72.3)$ & $125(43.4)$ & \multirow{3}{*}{0.034} \\
\hline & & & & & \\
\hline & & No & $31(27.7)$ & $163(56.6)$ & \\
\hline & \multirow[t]{2}{*}{ Wearing glasses } & Yes & $64(57.1)$ & $126(43.8)$ & \multirow[t]{2}{*}{0.047} \\
\hline & & No & 48(42.9) & $162(56.2)$ & \\
\hline
\end{tabular}


0.7. The collected data were analyzed in SPSS V. 19 using descriptive statistics and logistic regression analysis by considering $\mathrm{P}<0.05$.

\section{Results}

The Mean \pm SD age of the study participants was $78 \pm 8$ years. Most of them (52.5\%) were female and married $(69.5 \%)$ and were living with their husband/wife (54.3\%). Moreover, the majority of them had an educational level of lower than high school diploma (38.5\%), retired (48.3\%) owned an apartment (93.5\%), and living mostly in district $6(10 \%)$. Results also reported that 112 samples had experienced at least one fall (28\%) and 34\% more than one fall. Of 112 victims of falling, 64 (57.1\%) were female with a Mean \pm SD age of $80 \pm 7.5$ years. In terms of the location of the fall, $82.1 \%$ fell in the home and in the bathroom (23.4\%). The most injured area caused by the fall was pelvis $(26.8 \%)$, hand $(22.3 \%)$, and foot $(19.6 \%)$. Forty-nine $(43.8 \%)$ of seniors were admitted to hospital after the fall. Most of them stayed 1-5 days in the hospital.

According to the results in Table 1, regarding the relationship between having a fall and psychological risk factors, we can see that urinary incontinence $(\mathrm{P}=0.012)$, sleep disorders ( $\mathrm{P}=0.003)$, and fear of falling $(\mathrm{P}<0.001)$ had significant correlations with having a fall in seniors $(\mathrm{P}<0.05)$. Other psychological factors had no significant association with falling. Among medical risk factors, a history of surgery $(\mathrm{P}=0.037)$ had a significant correlation with fall $(\mathrm{P}<0.05)$, and others had no association with falling. Moreover, among sensory-motor risk factors, walking with difficulty $(\mathrm{P}<0.001)$, shaky hands and feet $(\mathrm{P}=0.008)$, knee pain $(\mathrm{P}<0.001)$, using mobility aids $(\mathrm{P}=0.034)$, and wearing glasses (vision impairment) $(\mathrm{P}=0.047)$ had significant relationships with falling at a significance level less than 0.05 , while wearing a hearing aid had a significant correlation with falling at a level of less than 0.01 . Other variables showed no significant correlation with falling. Furthermore, lifestyle and environmental risk factors showed no significant relationships with the fall of elderlies $(\mathrm{P}>0.05)$.

\section{Conclusion}

In our study, the prevalence of falling in elderly people was $28 \%$. In other studies, it was between $26 \%$ and $35 \%$ [14-20]. In this study, $43.8 \%$ of the falls resulted in the admission to the hospital. In Taiwan, fall-related hospital admission rate was $47.5 \%$ [21]. In line with other studies [22, 23 ], there was no significant relationship between gender and the falling rate of the elderly. However, a significant relationship was found between age and falling rate which is consistent with the findings of other studies [24-28]. No significant association was found between falling and other demographic factors such as marital status, education, residential area, and type of residence. Sensory-motor risk factors were the most effective factors in falling of elderly people. Rehabilitation measures for the elderly can reduce these risks and prevent their fall.

\section{Ethical Considerations}

Compliance with ethical guidelines

This study is an approved research project (registration code: 31287-38-01-95) supported by Deputy of Research, and Trauma and Surgery Research Center of Sina Hospital affiliated to Tehran University of Medical Sciences.

\section{Funding}

This study was financially supported by Trauma and Surgery Research Center of Sina Hospital affiliated to Tehran University of Medical Sciences.

\section{Authors' contributions}

Conceptualization: Ahmad Fayaz-Bakhsh, Faroogh Na'emani; Methodology and editing: Morad Esmaiil Zali, Faroogh Na'emani; Analysis, investigation, resources, and draft preparation: Zahra Sohrabi, Faroogh Na'emani; and Supervision and project administration: Ahmad FayazBakhsh, Morad Esmaiil Zali.

\section{Conflict of interest}

The authors declared no conflict of interest.

\section{Acknowledgments}

Authors would like to thank Dr. Vafa Rahimi Mogher, Deputy of Trauma and Surgery Research Center, and managers and staff of DAM Home treatment center affiliated to Academic Center for Education, Culture and Research of Tehran University of Medical Sciences for their valuable support and cooperation. 


\title{
بررسى فراوانى عوامل خطرآفرين سقوط در سالمندان تيرنده خدمات درمانى در منزل در سال بروسي فو147
}

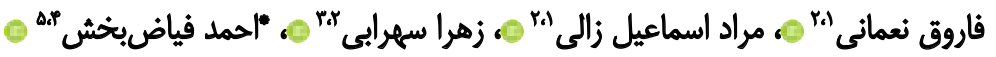 \\ 1- كروه علوم مديريت و اقتصاد بهداشته دانشكده بهداشت، دانشكاه علوم يزشكى وخدمات بهات بهداشتى برمانى تهران، تهران، ايران.

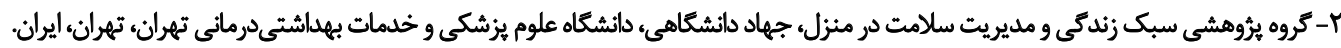

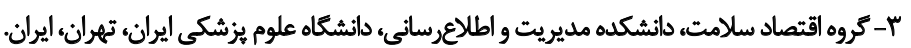

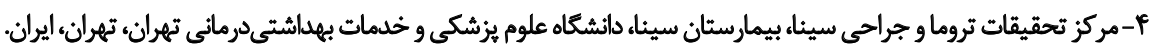

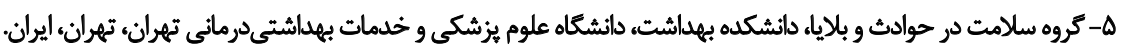

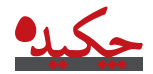

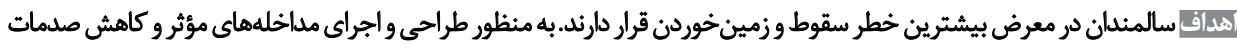

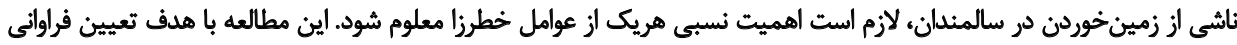

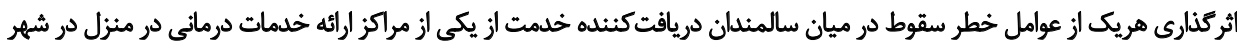
تههران انجام شد.

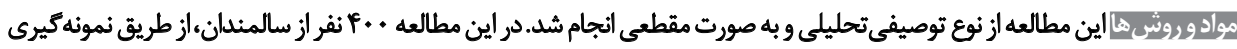

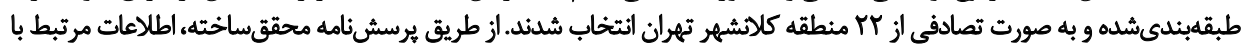

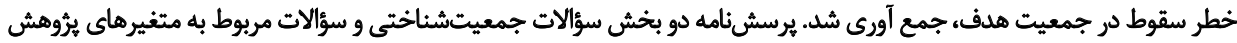

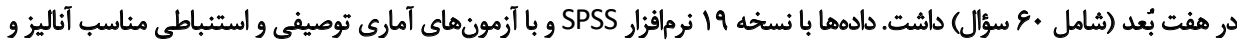
تجزيه و تحليل شد. تبرل

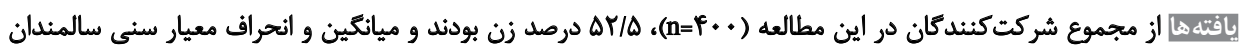

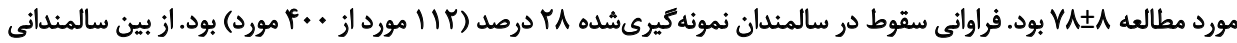

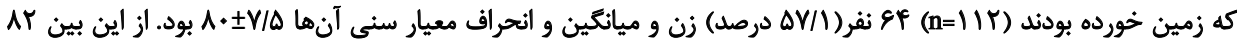

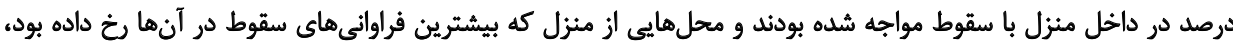

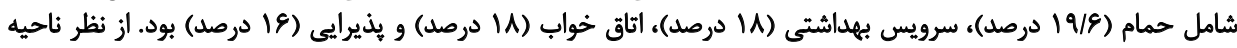

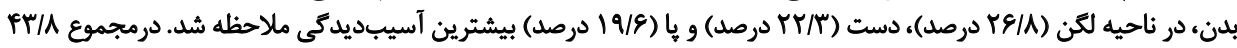

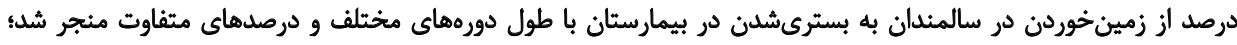

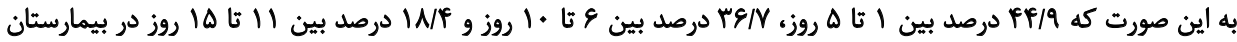

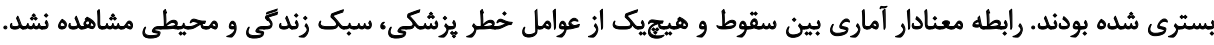

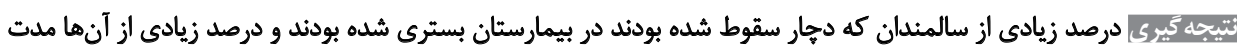

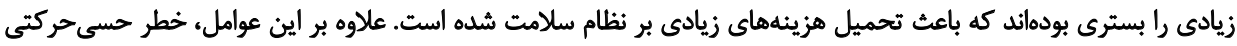

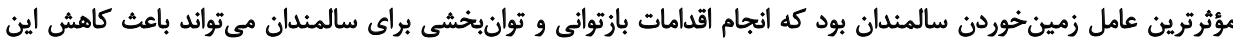

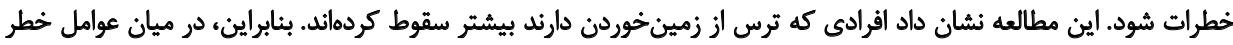

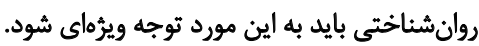

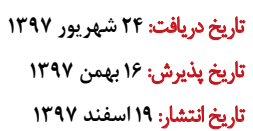

كليدوازوها:

زمين خوردن، عوامل

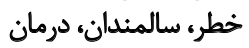
در منزله ايران 
كاهش فعاليت و تحرى سالمندان، به كاهش قداد قدرت

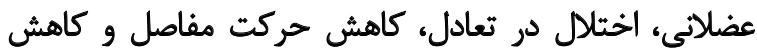

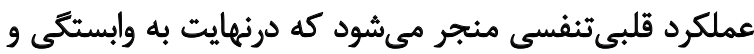

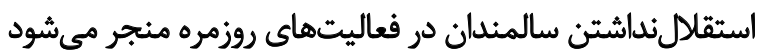

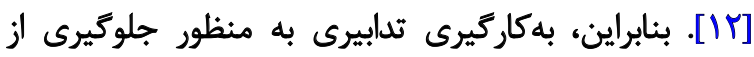

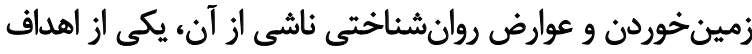

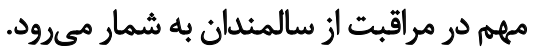
زمين خوردن سالمندان تأثيرات بسزاييى بر كيفيت زنداكى

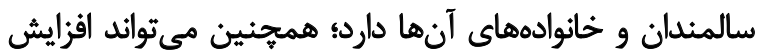

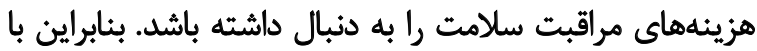

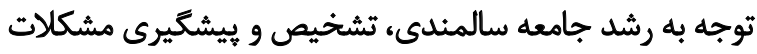

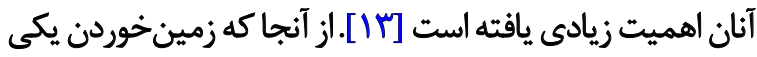

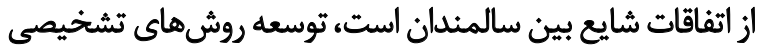

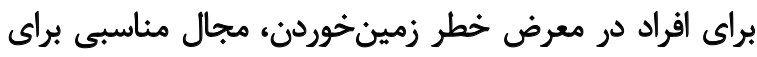

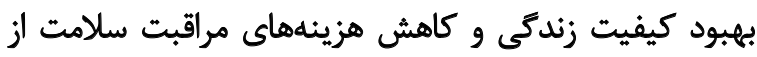

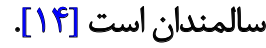

بر اين اساس يُروهشكران در اين مطالعه، در كام اول به دنبال

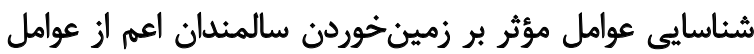

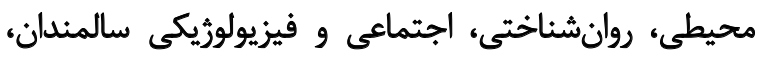

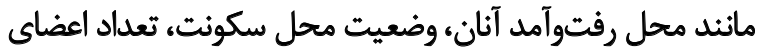

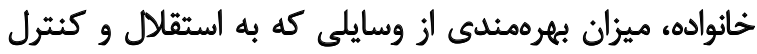

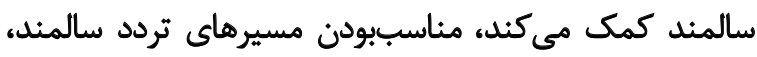

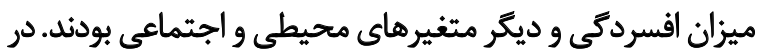

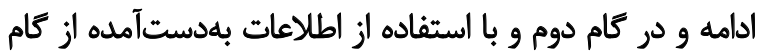

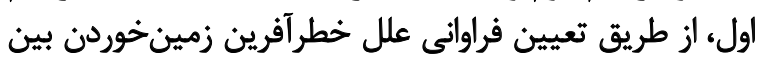

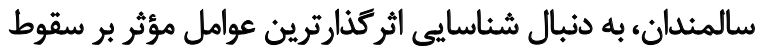

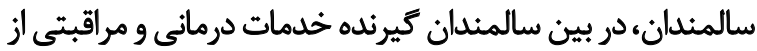
مركز درمان در منزل جهاد دانشكاهي (دم) بودند.

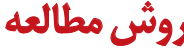

مطالعه حاضر مطالعهاى توصيفى تحليلى از نوع مقطعى است

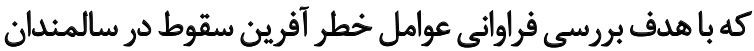

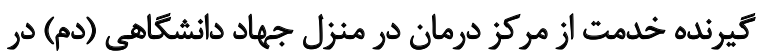

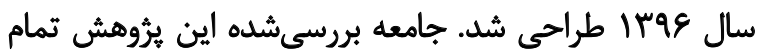

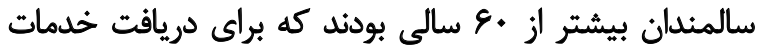

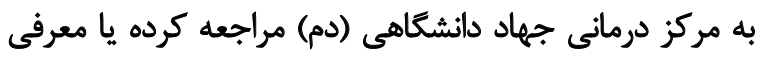

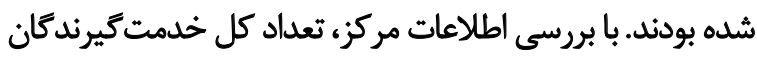

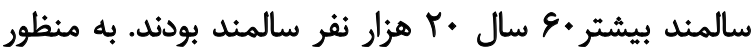

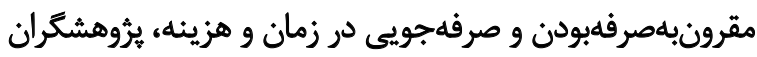

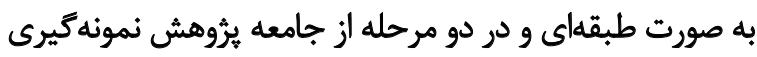

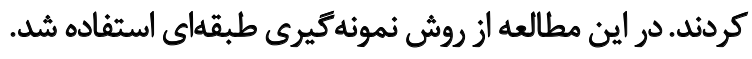

سالمندان با مشكلات عديدهاى در جامعه مواجهاند كه باعث

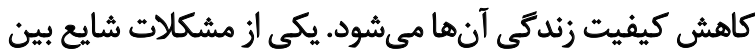

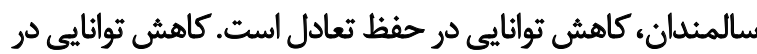

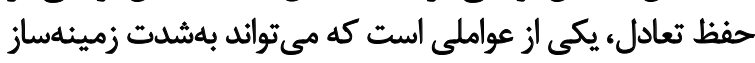

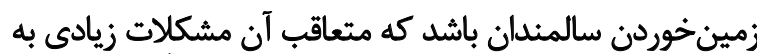

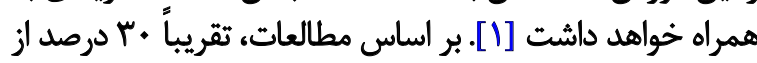

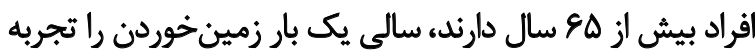

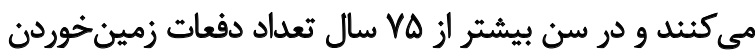

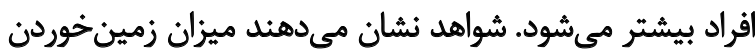

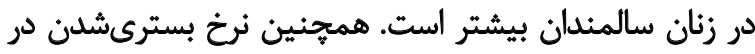

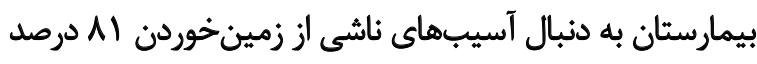

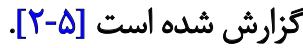

زمينخوردن كه يك حادثه به حساب ميى آيد، هزينهائ

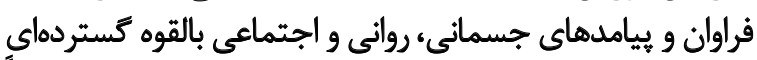

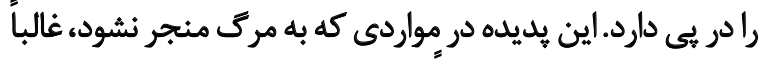

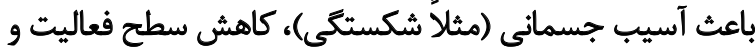

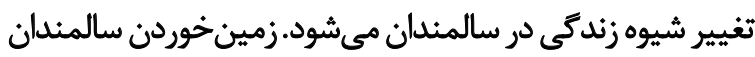

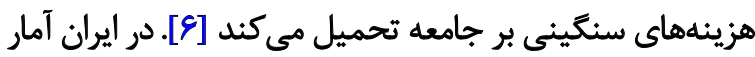

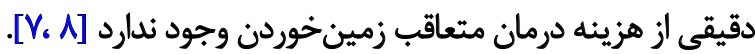

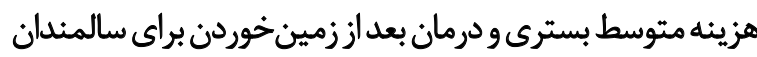

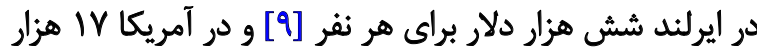

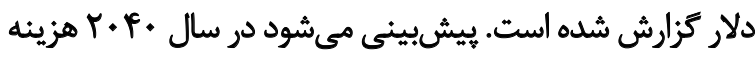

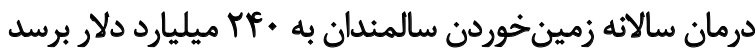

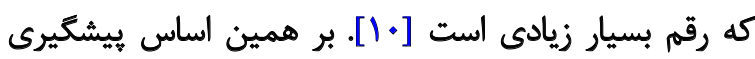

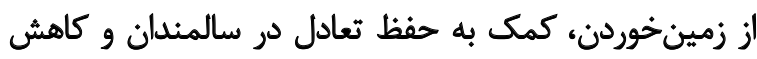

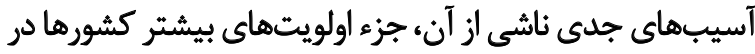
برنامهريزىهاى مربوط به مراقبت ازئ سالمندان است.

شايعترين نتيجه اجتماعى و روانى زمينخ زئوردن، ترس

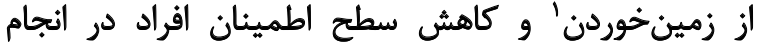

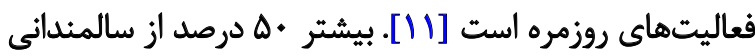

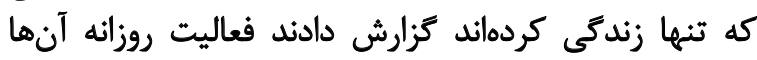

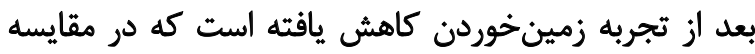

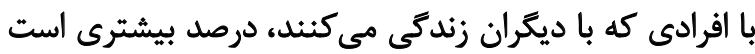

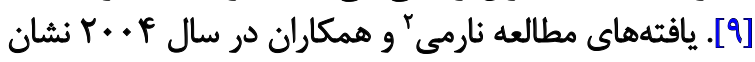

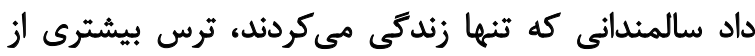

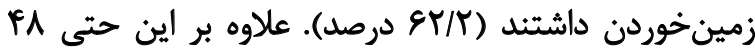

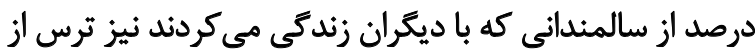

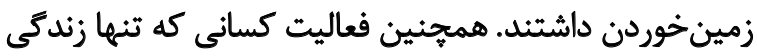
مى كردند، در مقايسه با كسانى كه با ديكران زندگى مينى مئى كردند،

1. Fear of falling

2. Nurmi 
خطر روانشناختى مؤثر بر سقوط سالمندان (19 كويه)، عوامل

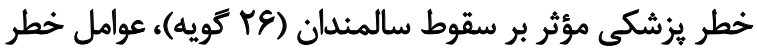

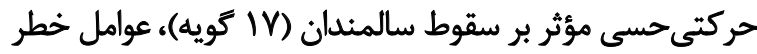

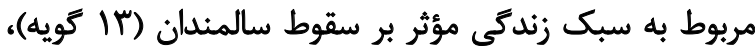

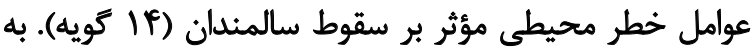

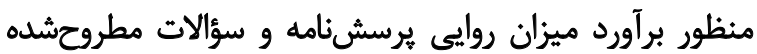

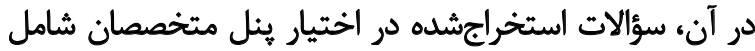

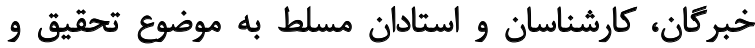
يرسشنامه قرار كرفت.

به دنبال اعمال نظر و اصلاحات خبركان و استادان، محققان

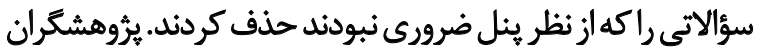

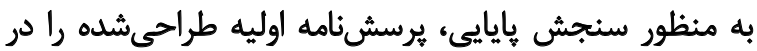

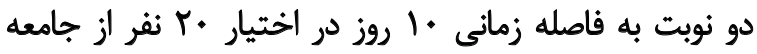

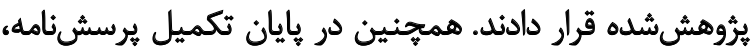

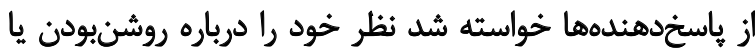

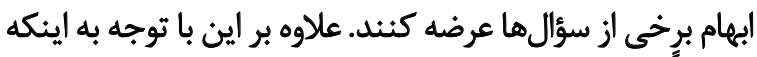

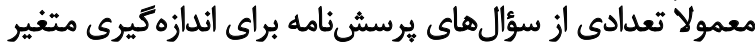

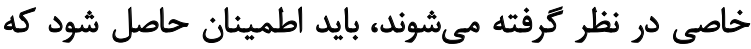

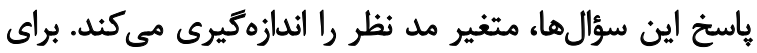

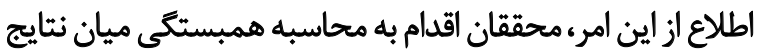

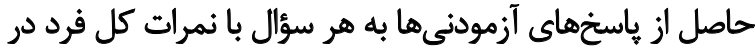

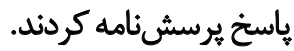

ضريب همبستكى زياد، ارتباط زياد ميان آنجه هر سؤال اندازه

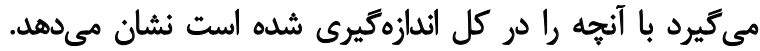

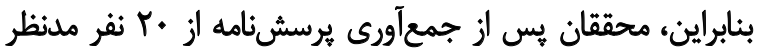

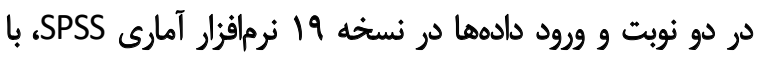

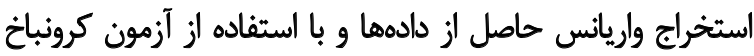

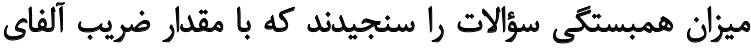

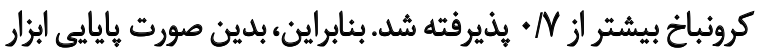

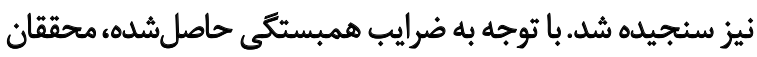

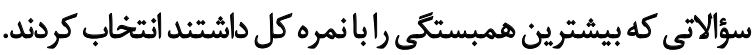

مبراى اين منظور موقعيت مكانى كيرندكان خدمات درماني

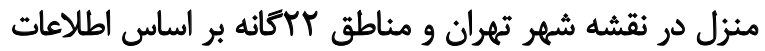

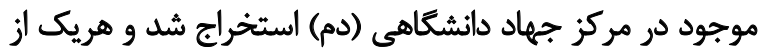

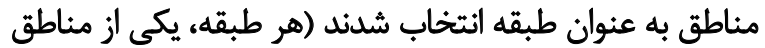

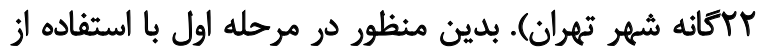

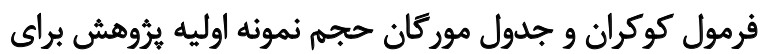

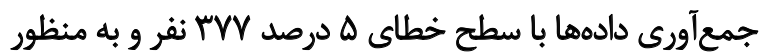

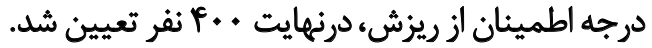

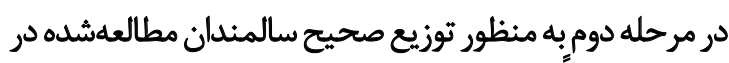

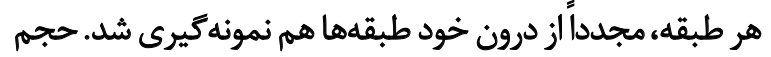

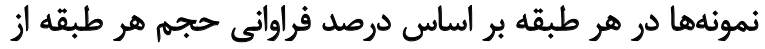

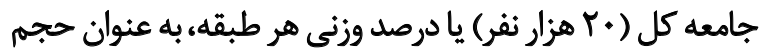

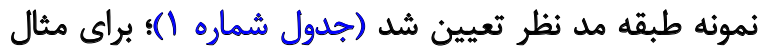

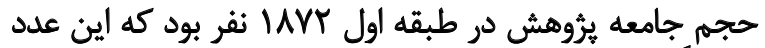

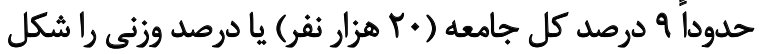

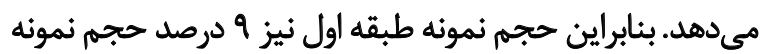

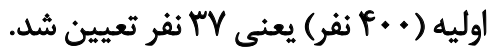

در ادامه به منظور طراحى برسشنامه عوامل خطر سقوط

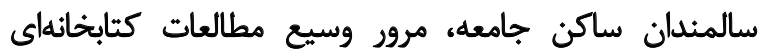

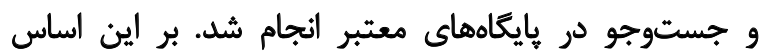

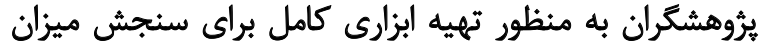

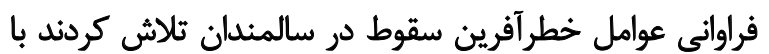

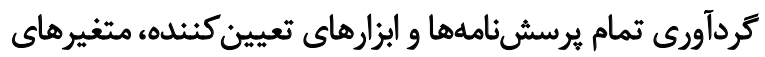

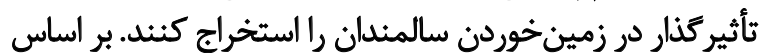

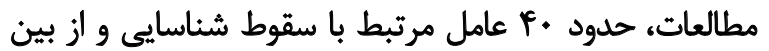

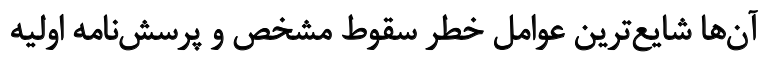

$$
\text { بر همين اساس تدوين شد. }
$$

در يايان يُروهشكران بر اساس نتايج بهدست آمده از مطالعات

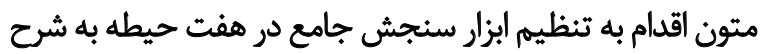

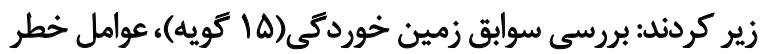

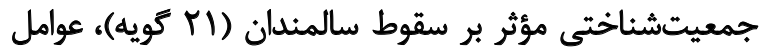

جدول ا. محاسبه حجم نمونه در هر طبقه

\begin{tabular}{|c|c|c|c|c|c|c|c|c|c|c|c|c|}
\hline 11 & 1. & 9 & $\Lambda$ & $\checkmark$ & 7 & $\bullet$ & \multicolumn{2}{|c|}{$\varepsilon$} & $r$ & Y & 1 & طبقهها (مناطق) \\
\hline $11 \pi$ & $9 .$. & PrI & 915 & TIMT & $r \cdot 1$. & $10 \%$ & \multicolumn{2}{|c|}{ If.. } & lear & IVAO & iAn & حجم جامعه (طبقه) \\
\hline Mr & $M$ & A & $M$ & rV & $r$ & $m$ & \multicolumn{2}{|c|}{ ru } & $m$ & re & $M$ & حجم نمونه \\
\hline جمع & rr & $M$ & r. & 19 & M & iv & 17 & 10 & 18 & ir & ir & طبقهها (مناطق) \\
\hline$r \ldots .$. & IfA & 108 & inr & $u$ & r... & ma & aro & $r \cdot r$ & apV & $9 \cdot 1$ & $1 \cdot \Delta s$ & حجم جامعه (طبقه) \\
\hline P.. & r & $r$ & $f$ & r & $p$ & $\Lambda$ & 11 & $\Lambda$ & 19 & M & M & حجم نمونه \\
\hline
\end{tabular}


همجنين ثيروهشكران با توجه به ماهيت سالمندى و مشكلات

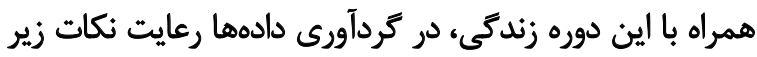

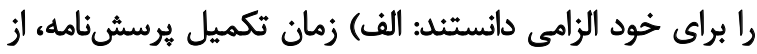

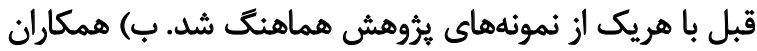

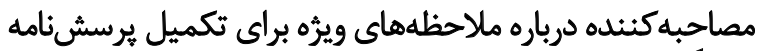

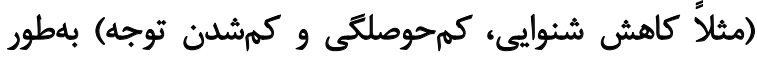

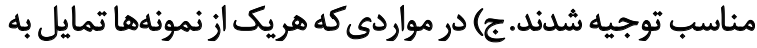

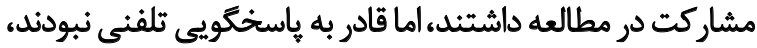

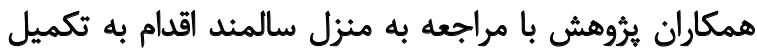

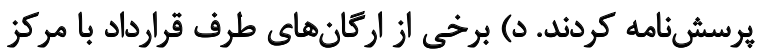

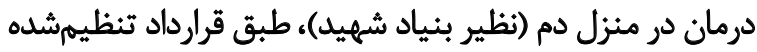

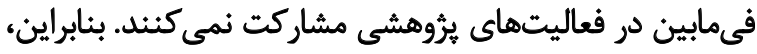

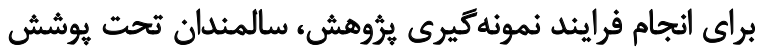

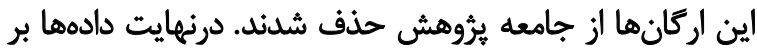

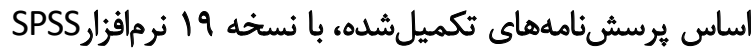

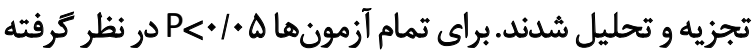

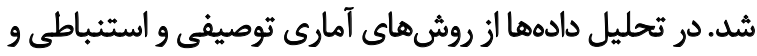
آزمون آمارى تحليل ركرسيون لجستيك استفاده شد.

يافتهها

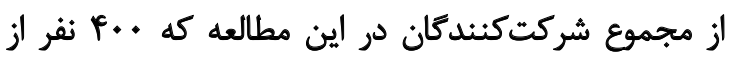

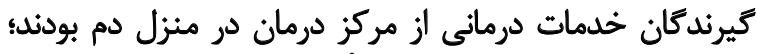

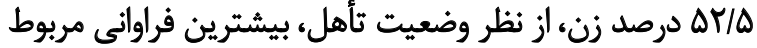

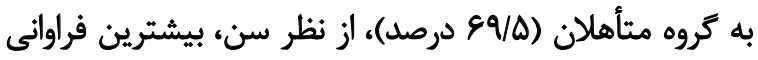

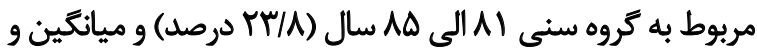

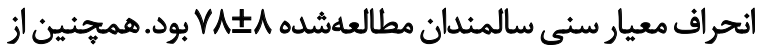

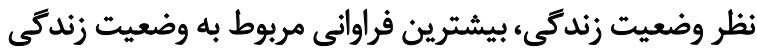

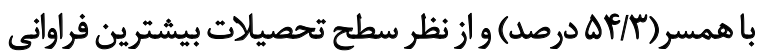

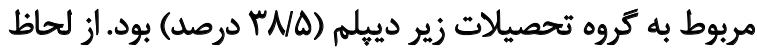

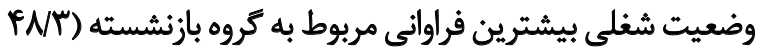

أز آنجا كه برسشنامه دوبخش سؤالات جمعيتشناختى و

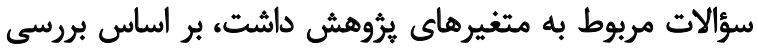

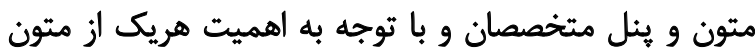

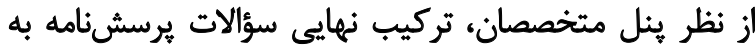

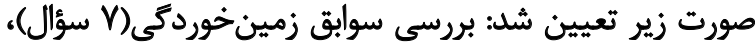

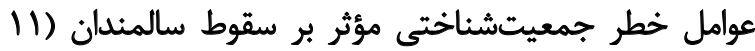

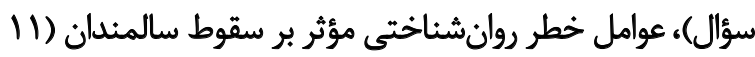

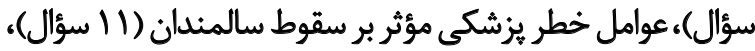

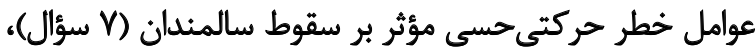

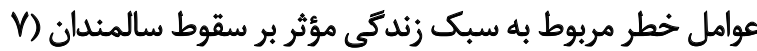

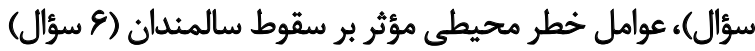

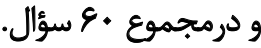

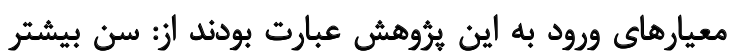

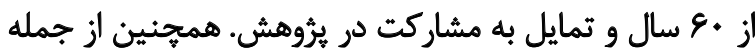

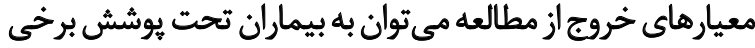

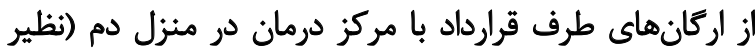

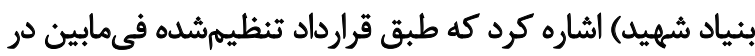
فعاليتهاى يرؤهشى مشاركت نمى كنيند.

يس از تهيه يرسشنامه، در مرحله دوم به منظور كردآورى

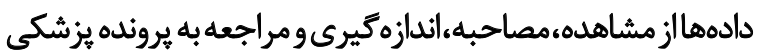

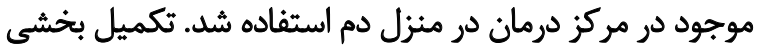

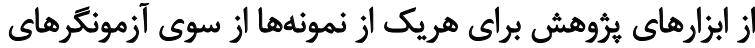

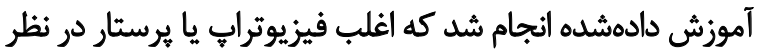

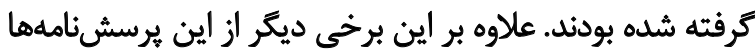

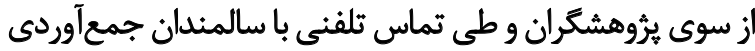

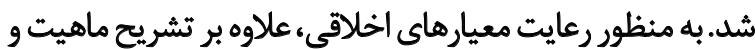

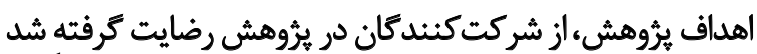

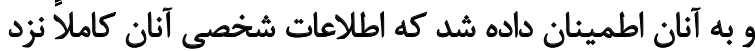

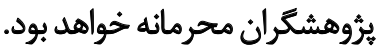

جدول r. فراوائى دفعات زمين خوردن

\begin{tabular}{|c|c|c|c|}
\hline درصد تجمعى & درصد & فراواتى & دفعات زمين خوردن \\
\hline eq & 88 & $n^{p}$ & 1 \\
\hline$N$ & $r$. & rr & $r$ \\
\hline q & V & $\Lambda$ & $r$ \\
\hline 9ท & $f$ & $\Delta$ & $F$ \\
\hline 99 & $r$ & $r$ & $\Delta$ \\
\hline \multirow[t]{2}{*}{$1+. \%$} & 1 & 1 & 8 \\
\hline & $1 .$. & IIr & كل \\
\hline
\end{tabular}

2 
جدول لَ. فراوانى محل زمين خوردن در منزل

\begin{tabular}{|c|c|c|c|c|}
\hline درصد تجمعى & درصد معتبر & درصد & فراوانى & متغير \\
\hline$r m / e$ & $\pi / T^{2}$ & $19 / 8$ & M & حمام \\
\hline$P F / N$ & $r / r$ & IV/a & r. & دستشويى \\
\hline$\Delta V / /$ & $e / f$ & $\Delta / \varphi$ & 8 & آشيزخائه \\
\hline$n \pi / r$ & $r / r$ & IV/9 & r. & اثاق خواب \\
\hline $91 / \infty$ & $19 / 1$ & $1 \% /$ & M & بذيرايى \\
\hline \multirow[t]{4}{*}{$1+0$} & NA & $v / 1$ & $\Lambda$ & ساير \\
\hline & $1 .$. & $\Lambda r / q$ & $9 f$ & كل ياسخدادهها \\
\hline & $\pi / F$ & $|9 /|$ & M & باستخداده \\
\hline & $r / / r$ & $1 .$. & $M r$ & كل \\
\hline
\end{tabular}

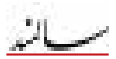

جدول F. فراوانى روزهاى بسترىشده در بيمارستان

\begin{tabular}{|c|c|c|c|}
\hline درصد تجمعى & درو & فراوانى & تعداد روزهاي بسترى \\
\hline$p+/ 9$ & $P \% / 9$ & rr & انا \\
\hline A)/8 & $M / V$ & M & ع أتا لروز \\
\hline \multirow[t]{2}{*}{$1 .+$} & WF & 9 & 11 \\
\hline & $1+$. & $p q$ & كل \\
\hline
\end{tabular}

فراواني مربوط به بسترىشدن در بيمارستان به مدت يك ثا ها روز

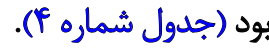

تحليل دادهاى جمعآورىشده به منظور بررسى ارتباط بين

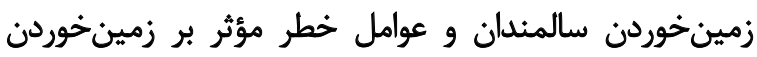

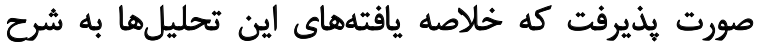
زير است: بررسى ارتباط بين زمينخوردين سالمندان و لاعوامل

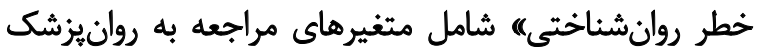

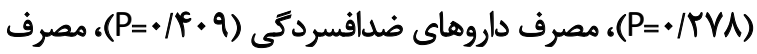

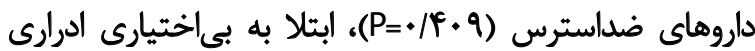

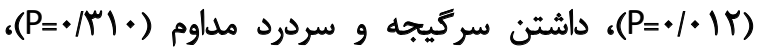

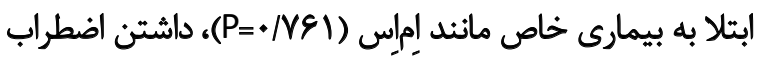
(P=•/MAF)

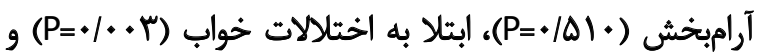

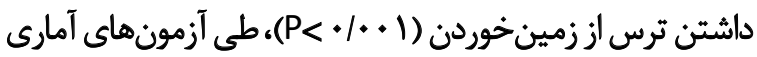

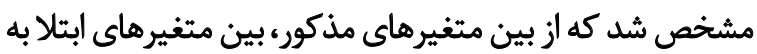

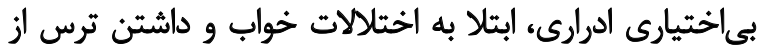
زمين خوردن با سقوط سالمندان در منزل ارتباط معنادارى وجيت إخود

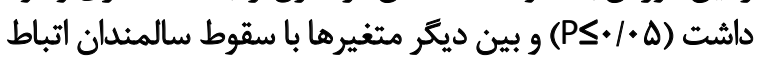

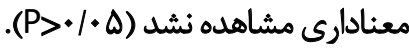

درصد) و از نظر نوع منزل بيشترين فراوانى مربوط به منزل

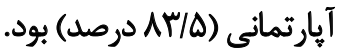

از نظر منطقه مسكونى بيشترين فراوانى مربوط به منطقائه

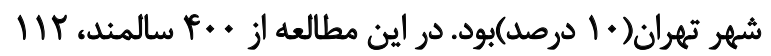

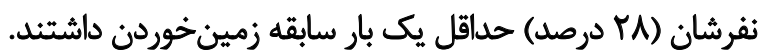

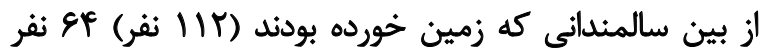

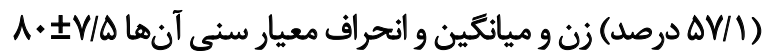

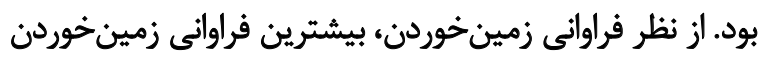

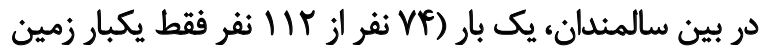

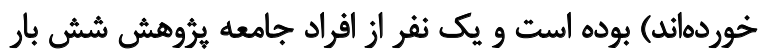

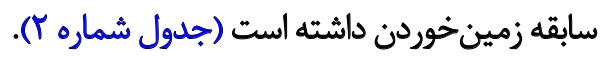

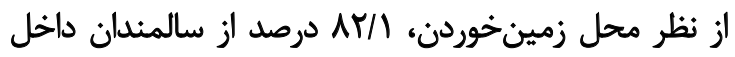

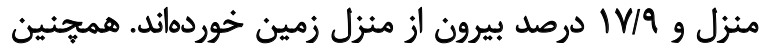

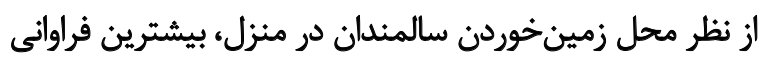

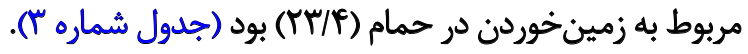
بين سالمندان اين مطالعه، بيشترين فراوانى ناحيه آسيبديده

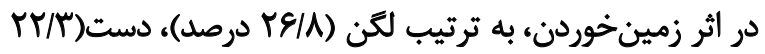

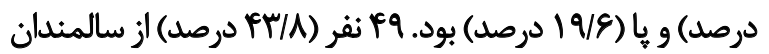
در اثر زمينخوردن، در بيمارستان بسترى شده بودند. بيشترين 
جدول ه. بررسى ارتباط بين زمينخوردن سالمندان و عوامل مؤثر بر آن به تفكيك نوع عامل خطر

\begin{tabular}{|c|c|c|c|c|c|}
\hline \multirow{2}{*}{ سطح معنى دارى } & \multicolumn{2}{|c|}{ آيا زمين خُورده اسث؟ } & \multirow{2}{*}{ هثنير } & \multirow{2}{*}{ مثغير } & \multirow{2}{*}{ عوامل موثر بر زمين خوردن } \\
\hline & خير (د,صد) & بله (درصد) & & & \\
\hline \multirow{2}{*}{.1 .14} & $1 . \Delta(r e / \Delta)$ & $R(\Delta F / N)$ & بله & \multirow{2}{*}{ ابتلا به بي اختيارى ادرارى } & \multirow{6}{*}{ عوامل خطر روانشناختى } \\
\hline & $\operatorname{Ar}\left(\varepsilon^{\prime} / \Delta\right)$ & $F q(F+/ V)$ & خير & & \\
\hline \multirow{2}{*}{+4} & $\operatorname{IrV}(r e / 1)$ & $V *(\varepsilon 8 / 1)$ & بله & \multirow{2}{*}{ ابتلا به اختلالات خواب } & \\
\hline & $|8|(\Delta \Delta / 9)$ & $r \wedge(m r / q)$ & خير & & \\
\hline \multirow{2}{*}{$\%+1$} & $1 \cdot \Lambda(F / / \Delta)$ & $q+(\Lambda \cdot / \pi)$ & بله & \multirow{2}{*}{ داشتن ترس از زمين خوردن } & \\
\hline & $M+(T V / \Delta)$ & $r r(19 / V)$ & خير & & \\
\hline \multirow{2}{*}{$\% \mathrm{rr}$} & $\operatorname{LAN}(\Delta P / q)$ & $r(\mathscr{R} / \mathrm{M})$ & بله & \multirow{2}{*}{ داشتن سابقه عمل جراحي } & \multirow{2}{*}{ عوامل خطر هيزشكى } \\
\hline & $\mid r \cdot(f \Delta / 1)$ & $F \cdot(r \Delta / V)$ & خير & & \\
\hline \multirow[t]{2}{*}{$.1 . .1$} & $119(9 / / m)$ & Ar $\left(V^{\prime} / 1\right)$ & بله & \multirow{2}{*}{ راهرقتن با مشكل } & \\
\hline & $\operatorname{leq}(\Delta N Y)$ & $r q(r \Delta / q)$ & خير & & \\
\hline \multirow{2}{*}{$\% 1$} & $9 \Delta(\Pi \pi / \bullet)$ & $g(\Delta F / \Delta)$ & بله & \multirow{2}{*}{ داثشتن لرزش دست و ها } & \\
\hline & $197(8 \times 1 \cdot)$ & $\Delta)(f \Delta / \Delta)$ & خير & & \\
\hline \multirow[t]{2}{*}{$.1 .+1$} & $\operatorname{Irr}(\varphi \otimes / \Lambda)$ & $q(\Lambda \Delta / V)$ & بله & \multirow{2}{*}{ داشتن ناراحتى مفاصل زانو } & عواهل خطر \\
\hline & $\Delta \Delta E(\Delta F / M)$ & $\mid f(\mid f / M)$ & خير & & حسى حركتي \\
\hline \multirow{3}{*}{$.1 . m$} & $\operatorname{Ira}(r+T / F)$ & $\lambda I(N / T)$ & بله & \multirow{3}{*}{ استفاده أز وسايل كمكىركتى } & \\
\hline & & & & & \\
\hline & $1 E(\Delta E / P)$ & $M(T V / V)$ & خير & & \\
\hline \multirow{2}{*}{$.1 . P V$} & $\operatorname{ME}(\boldsymbol{M T} / \mathrm{A})$ & $\operatorname{sf}(\Delta V / 1)$ & بله & \multirow{2}{*}{ (أشتفاده از عينكى بينايي) } & \\
\hline & $\operatorname{ler}(\Delta \& / Y)$ & $F A(F Y / Q)$ & خير & & \\
\hline
\end{tabular}

$(\mathrm{P} \leq \cdot 1 \cdot \Delta)$

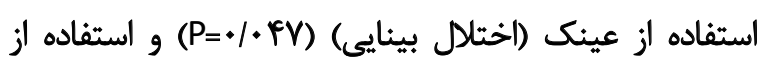

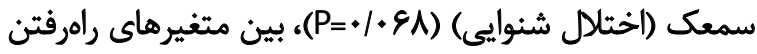

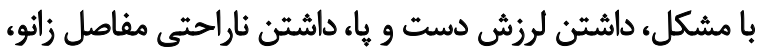

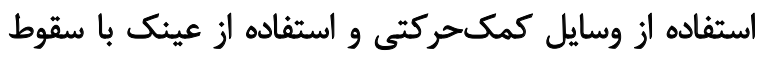

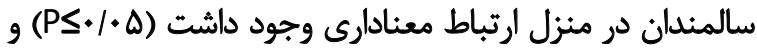
بين ديكر متغيرها با سقوط سالمندان ارتباط مناط معناداري مشارد مشاهده نشد (P> P (P) (جدول شماره Q ه).

بين "اعوامل خطر سبك زندكى)" شامل متغيرهاى مصرف

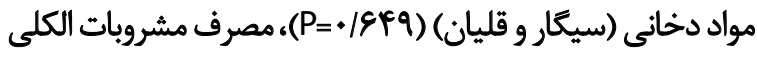
(P=//A•V)

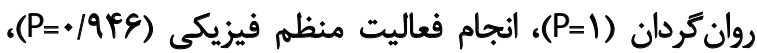

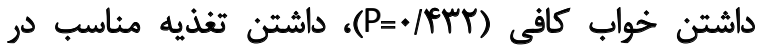

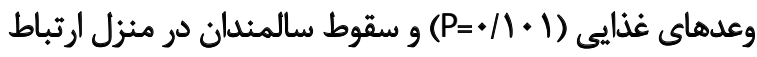

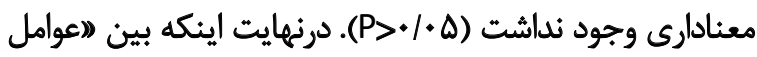

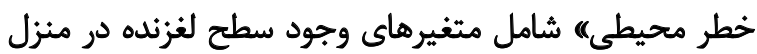

همجنين از بين "(عوامل خطر يزشكى) شامل متغيرهاى

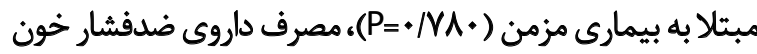

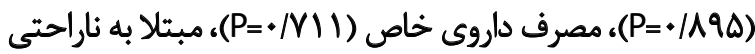

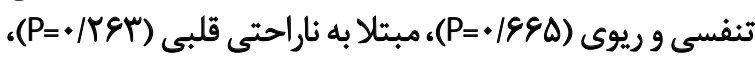

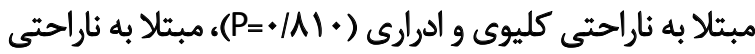

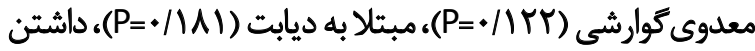

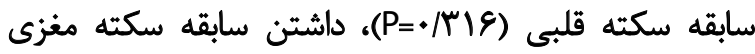

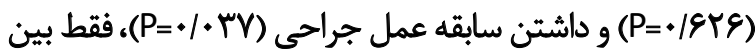

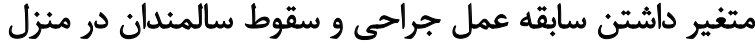

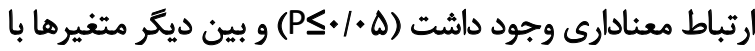

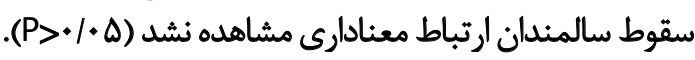
از بين " عوامل خطر حسى حركتى) شامل متغيرهاى راهرفتن

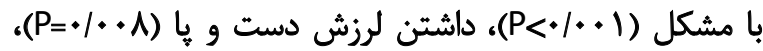

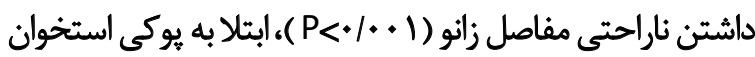

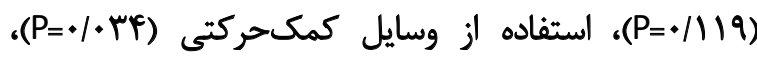


در ارتباط با متغير سن، مطالعه حاضر نشان داد بين سالمندان

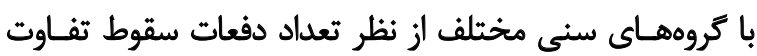

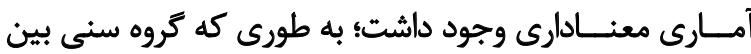

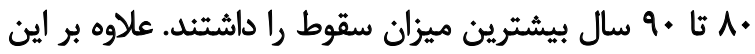

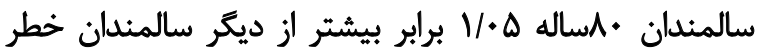

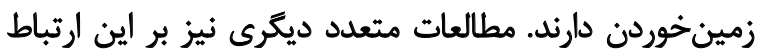

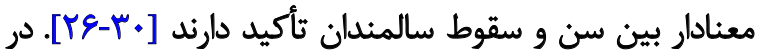

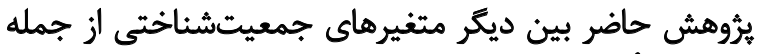

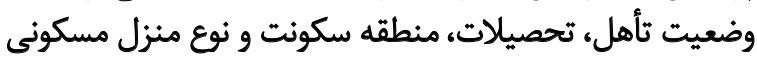
ارتباط معنادارى مشاهده نشد.

در مطالعه حاضر عوامل خطر مؤثر بر زمين خوردن سالمندان،

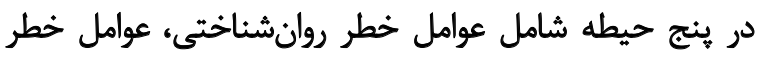

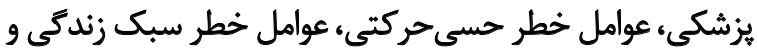

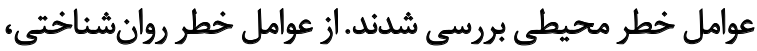

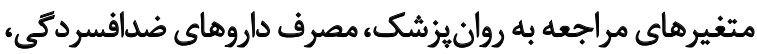

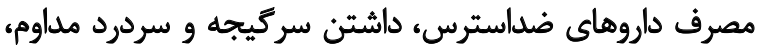

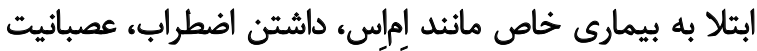

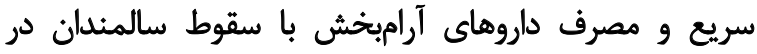

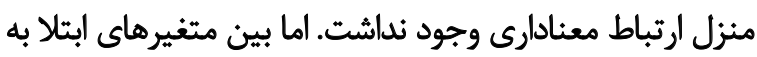

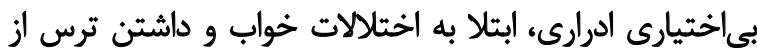

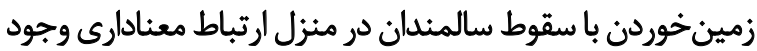

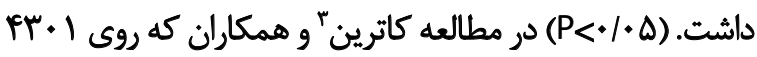

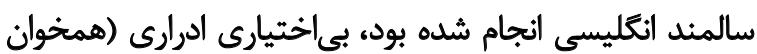

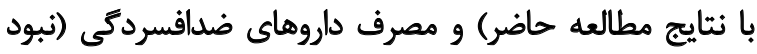

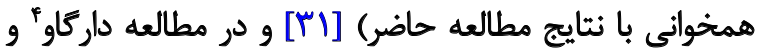

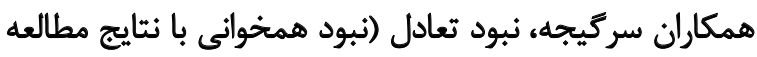

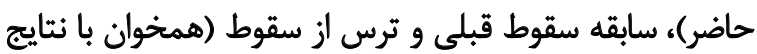

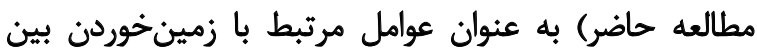

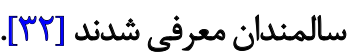

همجنين در ارتباط با ترس از زمينخوردن مطالعات نشان

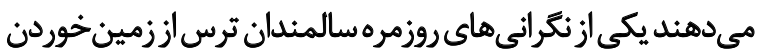

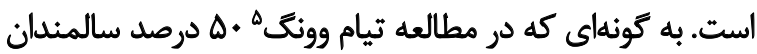

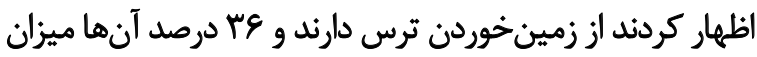

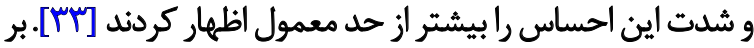

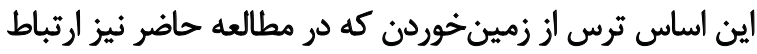

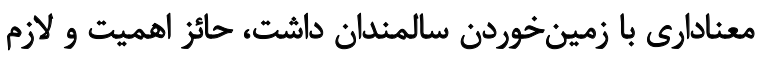

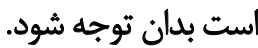

از عوامل خطر يزشكى، فقط متغير داشتن سابقه عمل

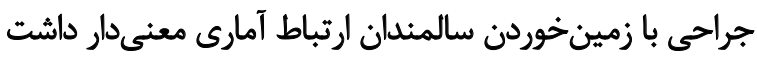

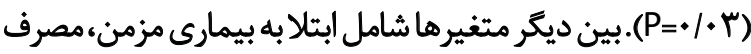

\section{Catharine}

4. Dhargave

5. Thiamwong
(P=•/AVF)

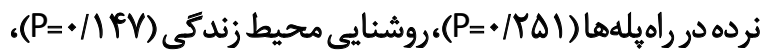

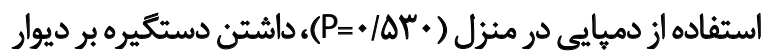

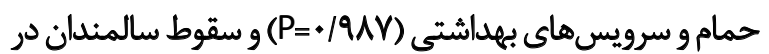

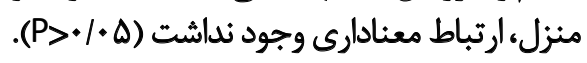

ث

در اين مطالعه كه با هدف بررسى فراوانى عوامل خطرآفرين

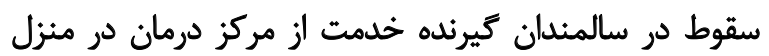

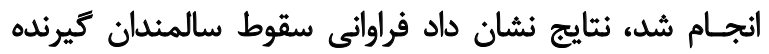

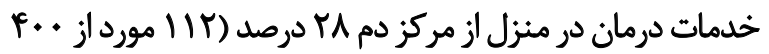

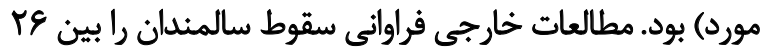

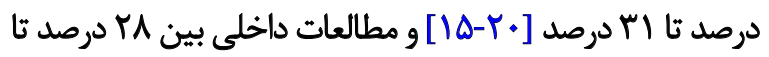

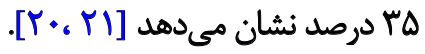

علاوه بر شيوع زمين خوردن بين سالمندان، يكى ديكر از نتايج

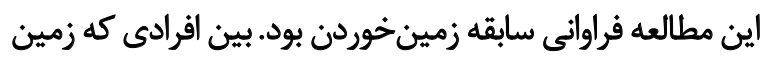

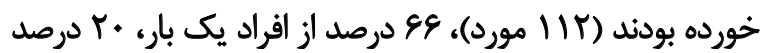

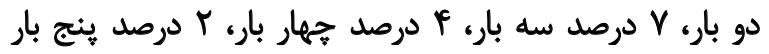

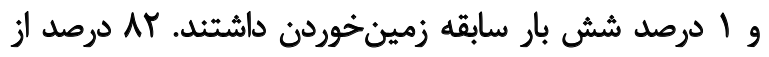

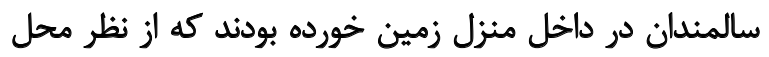

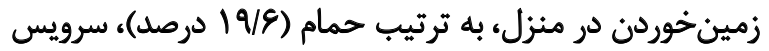

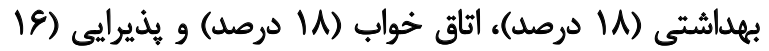

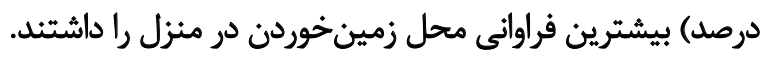

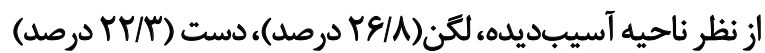

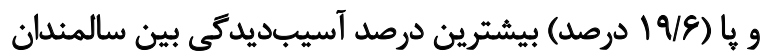
زمين خورده را داشتئد.

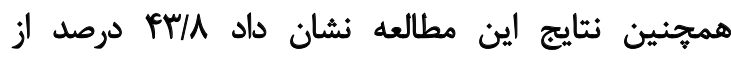

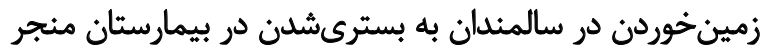

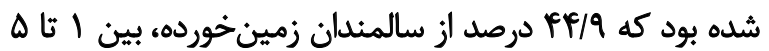

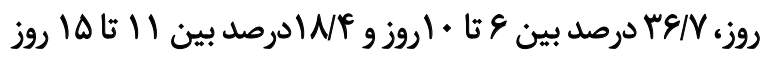

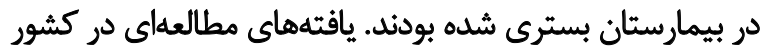

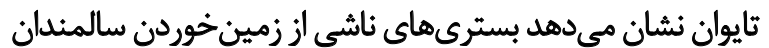

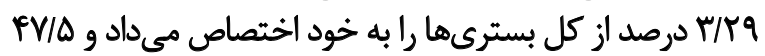

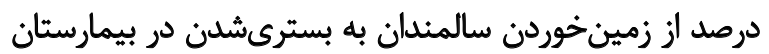

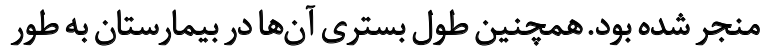

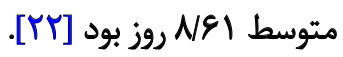

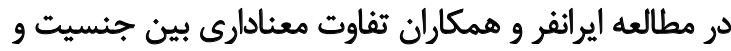

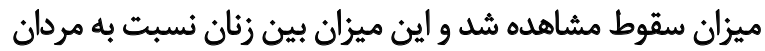

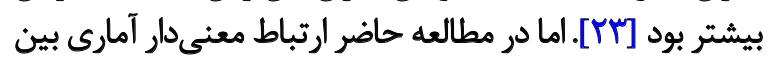

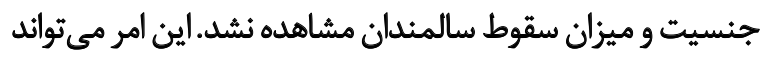

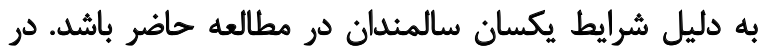

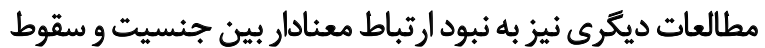

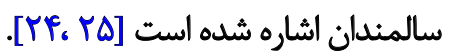


اين هزينههاى زيادى را بر نظام سلامت وارده كرده است. علاوه

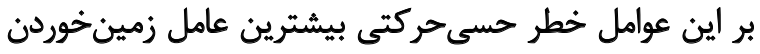

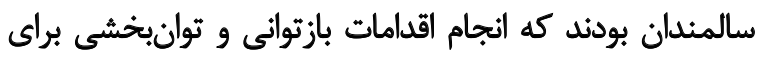

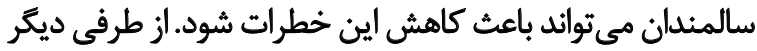

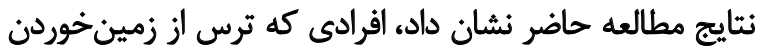

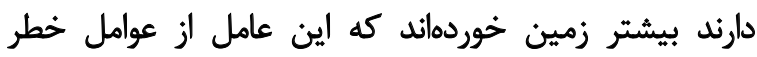
روانشناختى، نيازمند توجه ويروماى است.

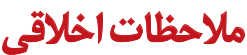

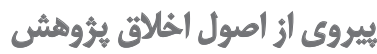

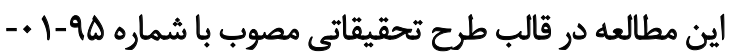

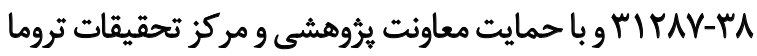
و جراحى بيمارستان سينا دانشكاه علوم يزشكى تهران انجام شدر.

$$
\text { مامي مالى }
$$

مطالعه حاضر با حمايتهاى مالى مركز تحقيقات تروما و بئي

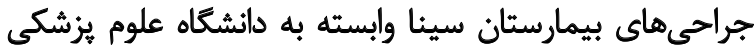
تهران انجام شده است.

$$
\text { مشاركت نويسند مكان }
$$

طراحى و مفهومسازى: احمد فياض بخش، فاروق نعمانى؛

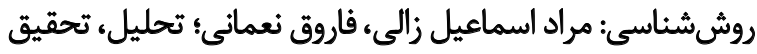

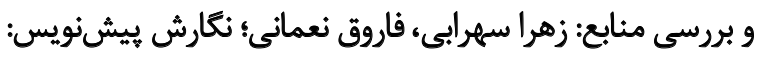

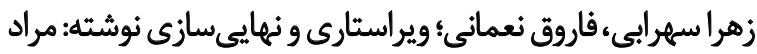

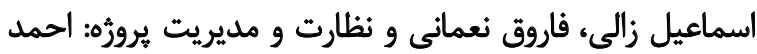

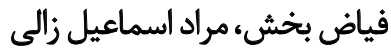

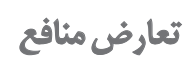

بنا به اظهار نويسندگان، در اين مقاله هيجزونه تعارض منافعى

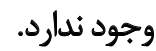

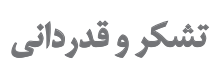

تويسند توان بر خود لازم مي دانيد از حمايتهاي مركز تحقيقات

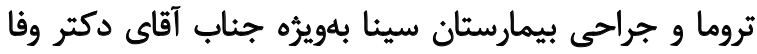

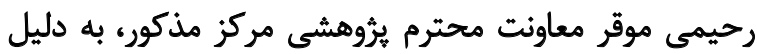

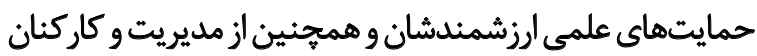

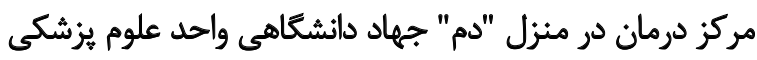

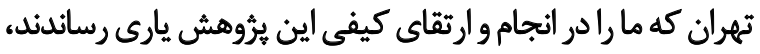
مراتب تشكر صميمانه خود را العلام كنيد.
داروى ضدفشار خون، مصرف داروى خاص، ابتلا به ناراحتى

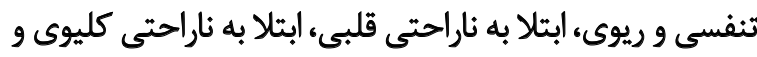

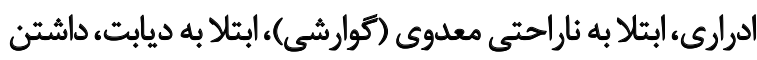

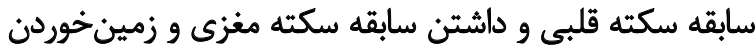

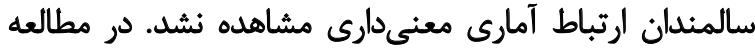

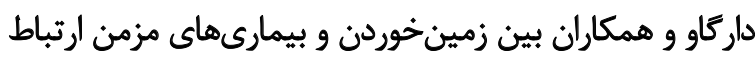

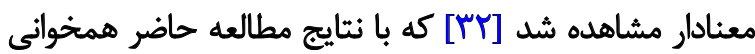

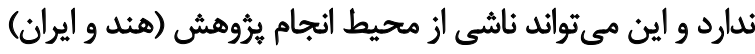

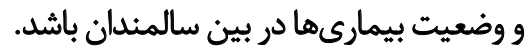

از عوامل خطر حسى دركتى متغيرهاى راهرفتن با مشكل

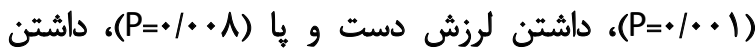

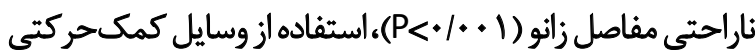

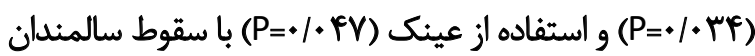
در منزل ارتباط آمارى معنادارى وجود داشين.

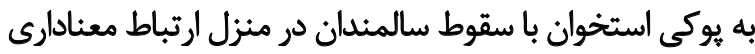

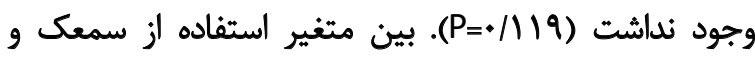

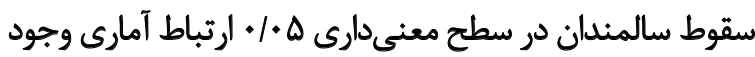

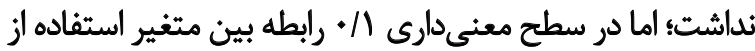

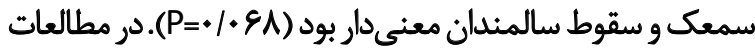

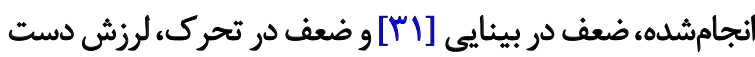

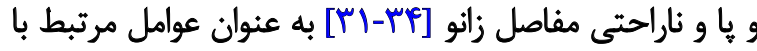
زمين وخوردن سالمندان شناسايى شدهاند.

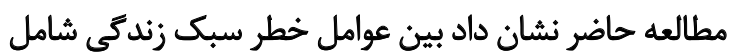

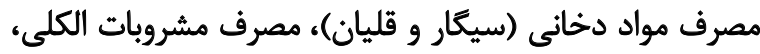

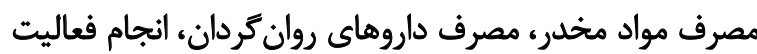

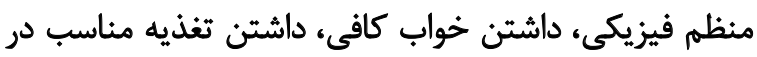
وعدهاى غذايى و سقوط سالمندان در منزل ارتباط داطن معنادارى

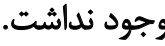

در اين مطالعه بين عوامل خطر محيطى شامل وجود سطح نورج

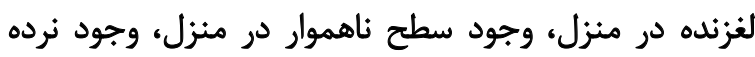

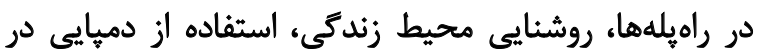

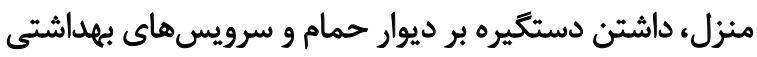

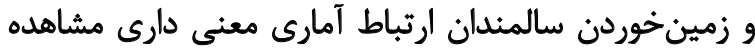

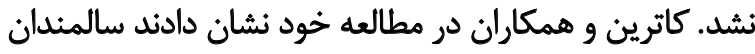

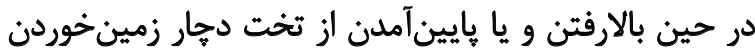

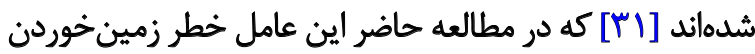
ارزيابى نشده بود.

\section{نتيجه كَيرى نهايي}

با توجه به نتايج مطالعه حاضر بهكاركيرى اقداماتى براى

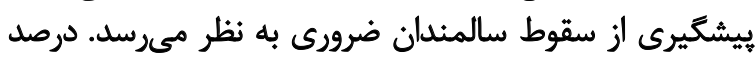

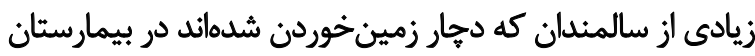
بسترى شدهاند و درصد زيادى، مدت زيار زيادى را بسترى بودين شداند در بيماند كه 


\section{References}

[1] Rasel M, Ardalan A. [The future of ageing and its health care costs: A warning for health system (Persian)]. Iranian Journal of Ageing. 2007; 2(2):300-5.

[2] Masud T, Morris RO. Epidemiology, of falls. Age and Ageing. 2001; 30(uppl_4):3-7. [DOI:10.1093/ageing/30.suppl_4.3] [PMID]

[3] Vitry AI, Hoile AP, Gilbert AL, Esterman A, Luszcz MA. The risk of falls and fractures associated with persistent use of psychotropic medications in elderly people. Archives of Gerontology and Geriatrics. 2010; 50(3):e1-e4. [DOI:10.1016/j.archger.2009.04.004] [PMID]

[4] Wu S, Keeler EB, Rubenstein LZ, Maglione MA, Shekelle PG. A cost-effectiveness analysis of a proposed national falls prevention program. Clinics in Geriatric Medicine. 2010; 26(4):751-66. [DOI:10.1016/j.cger.2010.07.005] [PMID]

[5] Adam SH, Eid HO, Barss P, Lunsjo K, Grivna M, Torab FC, et al. Epidemiology of geriatric trauma in United Arab Emirates. Archives of Gerontology and Geriatrics. 2008; 47(3):377-82 [DOI:10.1016/j.archger.2007.08.018] [PMID]

[6] Demura S, Yamada T, Kasuga K. Severity of injuries associated with falls in the community dwelling elderly are not affected by fall characteristics and physical function level. Archives of Gerontology and Geriatrics. 2012; 55(1):186-9. [DOI:10.1016/j.archger.2011.06.033] [PMID]

[7] Motlagh ME, Yazdani S, Tanjani PT. Elderly health profile in Islamic Republic of Iran. Tehran: Ministry of Health and Medical Education; 2014

[8] Aslankhani MA, Farsi A, Abdoli B, Zamani Sani H, Fathi Rezaei Z. Elderly identification at risk of falling, by equilibrium tests under dual duty conditions. Elder Journal. 2009; 4(12):7-15.

[9] Elliott S, Painter J, Hudson S. Living alone and fall risk factors in community-dwelling middle age and older adults. Journal of Community Health. 2009; 34(4):301-10. [DOI:10.1007/s10900-0099152-x] [PMID]

[10] Siracuse JJ, Odell DD, Gondek SP, Odom SR, Kasper EM, Hauser CJ, et al. Health care and socioeconomic impact of falls in the elderly. The American Journal of Surgery. 2012; 203(3):335-8. [DOI:10.1016/j.amjsurg.2011.09.018] [PMID]

[11] Zur O, Carmeli E, Himellfarb M, Berner YN. [Vestibular function, falls and hip fracture in elderly-a relationship study (Hebrew)]. Harefuah. 2004; 143(3):197-202. [PMID]

[12] Nurmi IS, Lüthje PM, Kataja JM. Long-term survival after falls among the elderly in institutional care. Archives of Gerontology and Geriatrics. 2004; 38(1):1-10. [DOI:10.1016/S01674943(03)00079-7]

[13] van Haastregt JC, van Rossum E, Diederiks JP, de Witte LP, Voorhoeve PM, Crebolder HF. Process-evaluation of a home visit programme to prevent falls and mobility impairments among elderly people at risk. Patient Education and Counseling. 2002; 47(4):301-9. [PMID]

[14] Bloch F, Gautier V, Noury N, Lundy JE, Poujaud J, Claessens YE, et al. Evaluation under real-life conditions of a stand-alone fall detector for the elderly subjects. Annals of Physical and Rehabilitation Medicine. 2011; 54(6):391-8. [DOI:10.1016/j.rehab.2011.07.962] [PMID]
[15] Halil M, Ulger Z, Cankurtaran M, Shorbagi A, Yavuz BB, Dede $\mathrm{D}$, et al. Falls and the elderly: Is there any difference in the developing world? A cross-sectional study from Turkey. Archives of Gerontology \& Geriatrics. 2006; 43(3):351-59. [Doi:10.1016/j. archger.2005.12.005]

[16] Chu LW, Chau AY, Chi I. Falls and subsequent health service utilization in community-dwelling Chinese older adults. Archives of Gerontology \& Geriatrics. 2008; 46(2):125-35. [DOI:10.1016/j. archger.2007.03.005]

[17] Stalenhoef PA, Diederiks JPM, Knottnerus JA, Kester AD, Crebolder HF. A risk model for the prediction of recurrent falls in community-dwelling elderly: a prospective cohort study. Journal of Clinical Epidemiology. 2002; 55(11):1088-94. [Doi:10.1016/ s0895-4356(02)00502-4]

[18] Swanenburg J, Bruin ED, Uebelhart D, Mulder T. Falls prediction in elderly people: A 1-year prospective study. Gait \& Posture. 2010; 31(3):317-21. [DOI:10.1016/j.gaitpost.2009.11.013]

[19] Morris M, Osborne D, Hill K, Kendig H, Lundgren-Lindquist B, Browning C, et al. Predisposing factors for accasional and multiple falls in older Australians who live at home. Australian Journal of Physiotherapy. 2004; 50(3):153-9. [Doi:10.1016/s00049514(14)60153-7]

[20] Coimbra AM, Ricci NA, Coimbra IB, Lavras Costallat LT. Falls in the elderly of the family health program. Archives of Gerontology \& Geriatrics. 2010; 51(3):317-22. [DOI:10.1016/j.archger.2010.01.010]

[21] Borhaninejad VR, Rashedi V, Tabe R, Delbari A, Ghasemzadeh $\mathrm{H}$. [Relationship between fear of falling and physical activity in older adults (Persian)]. Medical Journal of Mashhad University of Medical Sciences. 2015; 58(8):446-52.

[22] Hsiao YL, Chang CC, Chen CM. Profile of hospitalized elderly patients treated for falling. International Journal of Gerontology. 2012; 6(1):42-5. [DOI:10.1016/j.ijge.2012.01.019]

[23] Iranfar M, Ainy E, Soori H. [Fall epidemiology in the elderly residents of care centers in Tehran, 2010 (Persian)]. Iranian Journal of Ageing. 2013; 8(2): 30-8.

[24] Jafarian Amiri S, Zabihi A, Aziznejad Roshan P, Hosseini S, Bijani A. [Fall at home and its related factors among the elderly in Babol city, Iran (Persian)]. Journal of Babol University of Medical Sciences. 2013; 15(5):95-101.

[25] Corsinovi L, Bo M, Ricauda Aimonino N, Marinello R, Gariglio F, Marchetto C, et al. Predictors of falls and hospitalization outcomes in elderly patients admitted to an acute geriatric unit. Archives of Gerontology \& Geriatrics. 2009; 49(1):142-5. [DOI:10.1016/j.archger.2008.06.004] [PMID]

[26] Ungar A, Rafanelli M, IacomelliI, Brunetti MA, Ceccofiglio A, Tesi F, Marchionni N. Fall prevention in the elderly. Clinical Cases in Mineral and Bone Metabolism. 2013; 10(2):91-5. [PMID] [PMCID]

[27] Laessoe U, Hoeck HC, Simonsen O, Sinkjaer T, Voigt M. Fall risk in an active elderly population. Journal of Negative Results in BioMedicine. 2007; 6:2. [DOI:10.1186/1477-5751-6-2]

[28] Dionyssiotis Y. Analyzing the problem of falls among older people. International Journal of General Medicine. 2012; 5:805-13. [DOI:10.2147/IJGM.S32651]

[29] Almeida ST, Soldera CL, Carli GA, Gomes I, Resende TL. Analysis of extrinsic and intrinsic factors that predispose elderly 
individuals to fall. Revista da Associação Médica Brasileira. 2012; 58(4):427-33. [DOI:10.1016/S0104-4230(12)70224-5]

[30] Safavi Bayat Z, Zorriasatain F. [Determining risk factors associated with falling among elderly at residential care facilities in Tehran (Persian)]. Journal of Qazvin University of Medical Sciences. 2008; 11(4):66-70.

[31] Gale CR, Cooper C, Aihie Sayer A. Prevalence and risk factors for falls in older men and women: The English Longitudinal Study of Ageing. Age and Ageing. 2016; 45(6):789-94. [DOI:10.1093/ageing/afw129] [PMID] [PMCID]

[32] Dhargave P, Sendhilkumar R. Prevalence of risk factors for falls among elderly people living in long-term care homes. Journal of Clinical Gerontology and Geriatrics. 2016; 7(3):99-103.

[33] Thiamwong L, Suwanno J. Fear of Falling and Related Factors in a Community-based Study of People 60 Years and Older in Thailand. International Journal of Gerontology. 2017; 11(2):80-4. [DOI:10.1016/j.ijge.2016.06.003]

[34] Lopes KT, Costa DF, Santos LF, Castro DP, Bastone AC. Prevalence of fear of falling among a population of older adults and its correlation with mobility, dynamic balance, risk and history of falls. Brazilian Journal of Physical Therapy. 2009; 13(3):223-9. [DOI:10.1590/S1413-35552009005000026] 
This Page Intentionally Left Blank 